\title{
Coordinates, Conversions, and Kinematics for the Rochester Robotics Lab
}

\author{
Christopher M. Brown and Raymond D. Rimey
}

TR 259

August 1988

\begin{abstract}
This is a guide to coordinate systems, representations, and geometric relationships between them, for components of the Rochester Robotics Laboratory. The main entities at issue are the joint angles, location variables, and coordinate systems of the Puma, the camera angles and coordinate systems associated with the head, the spatial location of three-dimensional points, and the kinematic and inverse kinematic relationships between them. The robot-to-camera kinematic chain is described, conversions between homogeneous transformations and VAL location descriptions are provided, and inverse problems (camera angles to aim cameras at a $3-\mathrm{D}$ point given a robot configuration, binocular stereo calculations) are solved. Constants describing the robot head and sample robot description data structures are provided.
\end{abstract}

This work was supported by the DARPA U.S. Army Engineering Topographic Labs Grant No. DACA76-85-C-0001, and by the Air Force Systems Command, Rome Air Development Center, Griffiss AFB, NY, 13441-5700, and the Air Force Office of Scientific Research, Bolling AFB, DC, 20332, under Contract No. F30602-85-C-0008. This contract supports the Northeast AI Consortium (NAIC). The Xerox University Grants Program provided equipment used in the preparation of this paper. Peggy Meeker and Rose Peet produced the graphics. 


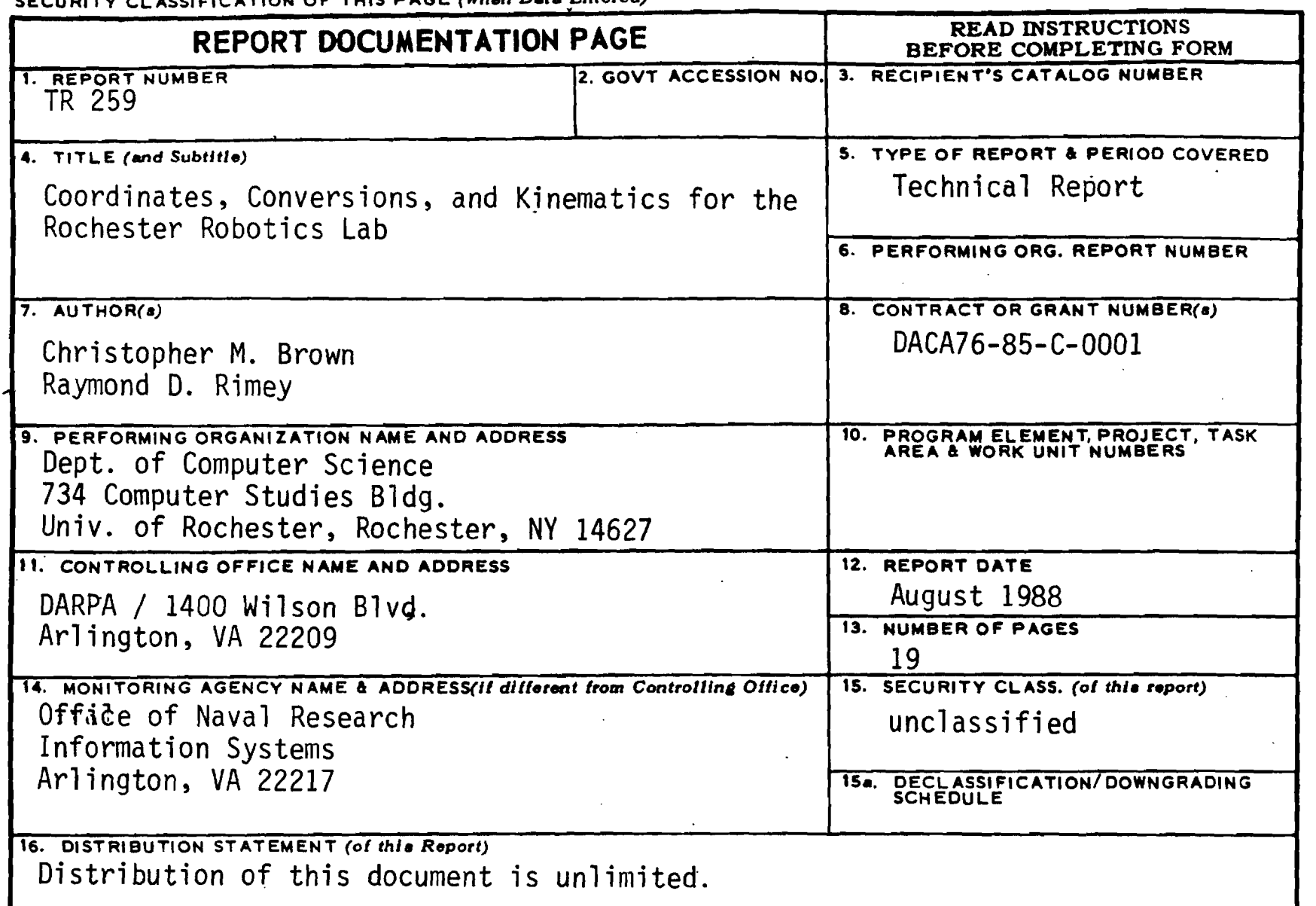

17. DISTRIBUTION STATEMENT (or the abstract ontered in Block 20, il difforent from Report)

18. SUPPLEMENTARY NOTES

19. KEY WORDS (Continue on reverse alde if noceseary and identlfy by block number)

Puma Róbot

Puma arm

Rochester "head"

coordinate systems

coord inate conversions

20. ABSTRACT (Continue on revorae alde if necosaery and ldentify by block number)

This is a guide to coordinate systems, representations, and geometric

relationships between them, for components of the Rochester Robotics Laboratory. The main entities at issue are the joint angles, location variables, and coordinate systems of the Puma, the camera angles and coordinate systems associated with the head, the spatial location of three-dimensional points, and the kinematic and inverse kinematic relationships between them. The robot-to-camera kinematic chain is described, conversions between homogeneous transformations and VAL location descriptions are provided, and inverse problems (camera angles

DD, FORM 1473 EDITION OF 1 NOV 65 IS OBSOLETE 


\section{ABSTRACT (Continued)}

to aim cameras at a 3-D point given a robot configuration, binocular stereo calculations) are solved. Constants describing the robot head and sample robot description data structures are. provided. 


\section{Purpose}

The purpose of this document is to relate elementary kinematic concepts and calculations to the University of Rochester Robotics Laboratory, with the aim of making certain aspects of the Puma arm and the two-camera head easier to use and understand. In using the robot to interact with the world, one quickly finds a potentially bewildering set of coordinate systems, angles, parameters, and state descriptions that must be related one to another in order to produce coherent robot actions and to answer common robotic questions. This document mainly concerns the definition and manipulation of coordinate systems. The semantics of the coordinates are such things as tool positions, camera orientations, and so forth.

Section two presents a short section on transformation notatation and properties, which should be read. There follows a glossary of scalars, vectors, and transformations we use later in the document, which can be skimmed and referred to as needed.

Section 3 defines some important robotic coordinate systems. LAB is the base coordinate system attached to the laboratory. TOOL describes the location of the robot head. FLANGE is another description for head location, but one more convenient for use with imaging operations.

Section 4 describes the model of the imaging process. Section 5 describes and defines the transformations along the chain of links that the Puma and the head embody.

There follow several sections, each one describing how to convert from one representation to another, or deriving a desired transformation or description from a specification. For example, it may be of interest to convert the TOOL coordinate system into the description used by VAL for robot location. An example of deriving an interesting configuration is to find the camera altitude and azimuth angles that the camera at a point in $(\mathrm{X}, \mathrm{Y}, \mathrm{Z})$ space.

There is room for expansion of this report, and an expanded version should be produced later when more is known. One obvious lack at present is the Jacobian calculations for the head -- at what rate to move the cameras to compensate continuously for continuous head motion and vice-versa.

\section{Transform Basics}

We follow [Paul 1981] and represent 3-space points as column homogeneous 4vectors or column Cartesian 3-vectors. Transforms (and coordinate systems, or CS's), are homogeneous $4 \times 4$ matrices. All transforms but the camera transform are rigid. Thus they denote a rigid rotation or translation or both. If both, then think of the rotation as being done before the translation. A transform $B$ operates on points expressed as column vectors to yield new points. A transform B represents a CS in that it can be thought of as four columns, three of which represent points at infinity and correspond to directions of the $X, Y$, and $Z$ axes of a CS, and the last of which represents a 3-space point and corresponds to the origin of the CS. Transforming (that is, multiplying) a CS by a transform just rigidly moves the CS in space. LAB is the identity transform, and transforming LAB by B yields B. Thus B cleverly represents a coordinate system and a transform that moves $\mathrm{LAB}$ to that coordinate system.

If $\vec{x}$ is a vector denoting a point in LAB coordinates, and A and B are transforms,

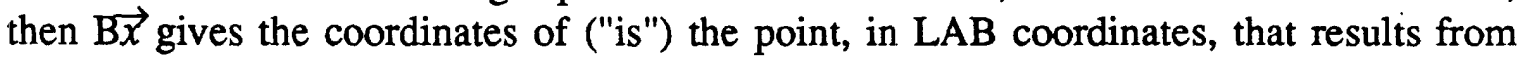


rotating and translating $\vec{x}$ by $\mathrm{B}$. $\mathrm{AB} \vec{x}$ is the point resulting from applying $\mathrm{B}$ to $\mathrm{x}$, then $\mathrm{A}$ to the result, where rotating and translating by $A$ is done with respect to the original LAB coordinate system. Alternatively, $\mathrm{AB} \overrightarrow{\mathcal{x}}$ means applying to $\vec{x}$ the transformation $\mathrm{A}$, followed by the transform B expressed in the frame A. is conceptualized as taking place in the coordinate system induced by all previous movements, the final transform is $A_{1} A_{2} \cdots A_{n}$ if 1 is the link connected to LAB. If $\vec{x}$ is a point in LAB and $B$ is a frame; then $\vec{x}$ expressed in $B$ is $B^{-1} \vec{x}$. Last, if $B$ takes LAB to the CS $B$ (its alibi aspect), then $B$ also is the transform from $B$ coordinates to $L A B$ coordinates (its alias aspect).

\subsection{Scalars}

VÂL expresses distance in mm, angìes in degrees. Parameters to our subroutines are expressed in radians, to save conversions. Since users often prefer degrees, our user interfaces (and this document) usually express angles in degrees.

Effective imaging system focal length (including digitizing effects). (Note that this number has nothing to do with the physical focal length of the lens).

s Camera aspect ratio: column spacing / row spacing.

$\phi \quad$ Camera platform altitude angle in radians.

$\theta \quad$ Generic azimuth angle.

$\theta_{L} \quad$ (Head's) Left Camera azimuth angle.

$\theta_{R} \quad$ Right Camera azimuth angle.

\subsection{Vectors}

Pixel coordinates in MaxVideo routines are expressed as (pixel offset in scanline, scanline), which corresponds to the "physical" (x,y) coordinate scheme used in the imaging model coordinate system, which has $\mathrm{Y}$ axis down and $\mathrm{X}$ axis to the right. In array indexing, however, the "natural" element-addressing scheme is Image[row][column], which has semantics $(y, x)$. Where this document uses pixel coordinates, they are expressed in the physical system rather than in the array-indexing system.

$\vec{x}$

$(\hat{x}, \hat{y})$

(O,A,T) "Orientation, Altitude, and Twist" angles describing the orientation of TOOL axes in terms of LAB. Like Euler angles but not (see below).

Loc $\quad(\mathrm{X}, \mathrm{Y}, \mathrm{Z}, \mathrm{O}, \mathrm{A}, \mathrm{T})$. A generic location $(\mathrm{X}, \mathrm{Y}, \mathrm{Z}$ position and orientation) used by VAL. May be relative. In this document we usually construe Loc to define the location of the TOOL CS in terms of LAB.

Head $\left(\phi, \theta_{L}, \theta_{R}\right)$, Camera rotations defining the head configuration.

JntAngs six angles defining the rotations of the robot links. Used by VAL software as "precision points". 


\subsection{Matrices, Coordinate Systems, and Transforms}

$S_{i j} \quad$ The $i^{\text {th }}$ row, $j^{\text {th }}$ column element of $\mathrm{S}$.

$\mathrm{A}_{i} \quad$ An "A Matrix", expressing the rigid transform induced by one link in a kinematic chain.

$\mathrm{T}_{j} \quad \mathrm{~A}$ transform induced by a kinematic chain. By definition, $T_{j}=\mathrm{A}_{1} \mathrm{~A}_{2} \cdots \mathrm{A}_{j}$.

Rot_x(a) Rotation around $X$ axis by angle a.

Rot_y(a) Similar

Rot_z(a) Similar

Trans $(x, y, z) \quad$ Translate by $x, y, z$.

LAB CS attached to the laboratory, defined below.

TOOL A user-definable coordinate system rigidly attached to joint 6 of the Puma.

NULLTOOL VAL's default value for TOOL. It corresponds to a relative location of $(\mathrm{X}, \mathrm{Y}, \mathrm{Z}, \mathrm{O}, \mathrm{A}, \mathrm{T})=(0,0,0,90,-90,0)$.

FLANGE CS convenient for head and camera calculations. Like another TOOL CS, it is rigidly attached to joint 6 . Defined in terms of NULLTOOL or $T_{6}$-relative to $T_{6}$ it has a Location of $(0,0,0,-180,0,-90)$ (see below).

C A perspective camera transform, not a rigid CS transform.

CamPos FLANGE transformed so its $\mathrm{Z}$ axis points along a camera's optic axis and its origin is at the front principal point of the lens. CamPosL and CamPosR are for left and right cameras.

PhysPixel Translates pixel coordinates so origin is upper left corner of pixel array, not its middle.

\section{Coordinate Systems and Robot Coordinates}

\subsection{LAB}

The Puma's internal representations assume that its first link is rigidly attached to a LAB coordinate system. VAL generally reports locations in LAB coordinates. The Puma's BASE coordinate system is usually synomynous with LAB. BASE may be changed by invoking the VAL BASE command, which allows translation of the $X, Y, Z$, origin and Z-rotation of BASE. An automatic $\mathrm{Z}$ rotation may be a good idea, since the Puma is bolted slightly askew.

At initialization, according to the manual, the origin of LAB is at the intersection of Joints 1 and 2. Certainly the origin is somewhere near the centerline of the Puma. Imagine you are looking in at the Puma through the window. Then in the default state, the $\mathrm{LAB} X$ axis points to your right parallel to the window, the $\mathrm{Y}$ axis is pointing away from you toward the far wall, and $\mathrm{Z}$ is up (Fig. 1). If all joint angles are 0 (JntAng $=\overrightarrow{0}$ ), TOOL $=$ NULLTOOL, and Head $=(0,0,0)=0$, the cameras are pointing away from you down TOOL (and LAB) Y. 


\section{2. $T_{6}$ and TOOL}

$T_{6}$ is a CS attached to the end of the last link of the robot. The TOOL CS is defined relative to $T_{6} . T_{6}$, TOOL, and FLANGE share a common origin. $T_{6}$ is only useful to understand the TOOL coordinate system. When JntAng $=\overrightarrow{0}$ (Fig. 1), $T_{6}$ has its $X$ axis pointing down (along $L A B-Z$ ), its $Y$ axis pointing along $L A B X$, and its $Z$ axis along LAB -Y (Fig. 1).

The TOOL coordinate system is a transformation of $T_{6}$, and is a primitive notion in VAL commands, which can often be expressed in TOOL coordinates. Upon initialization, TOOL $=$ NULLTOOL, which (Fig. 1) makes $T_{7}$ simply a translation of LAB if JntAng $=\overrightarrow{0}$. VAL reports the location of the TOOL CS in the form Loc $=$ $(\mathrm{X}, \mathrm{Y}, \mathrm{Z}, \mathrm{O}, \mathrm{A}, \mathrm{T})$. NULLTOOL corresponds to a relative location, with respect to $T_{6}$, of

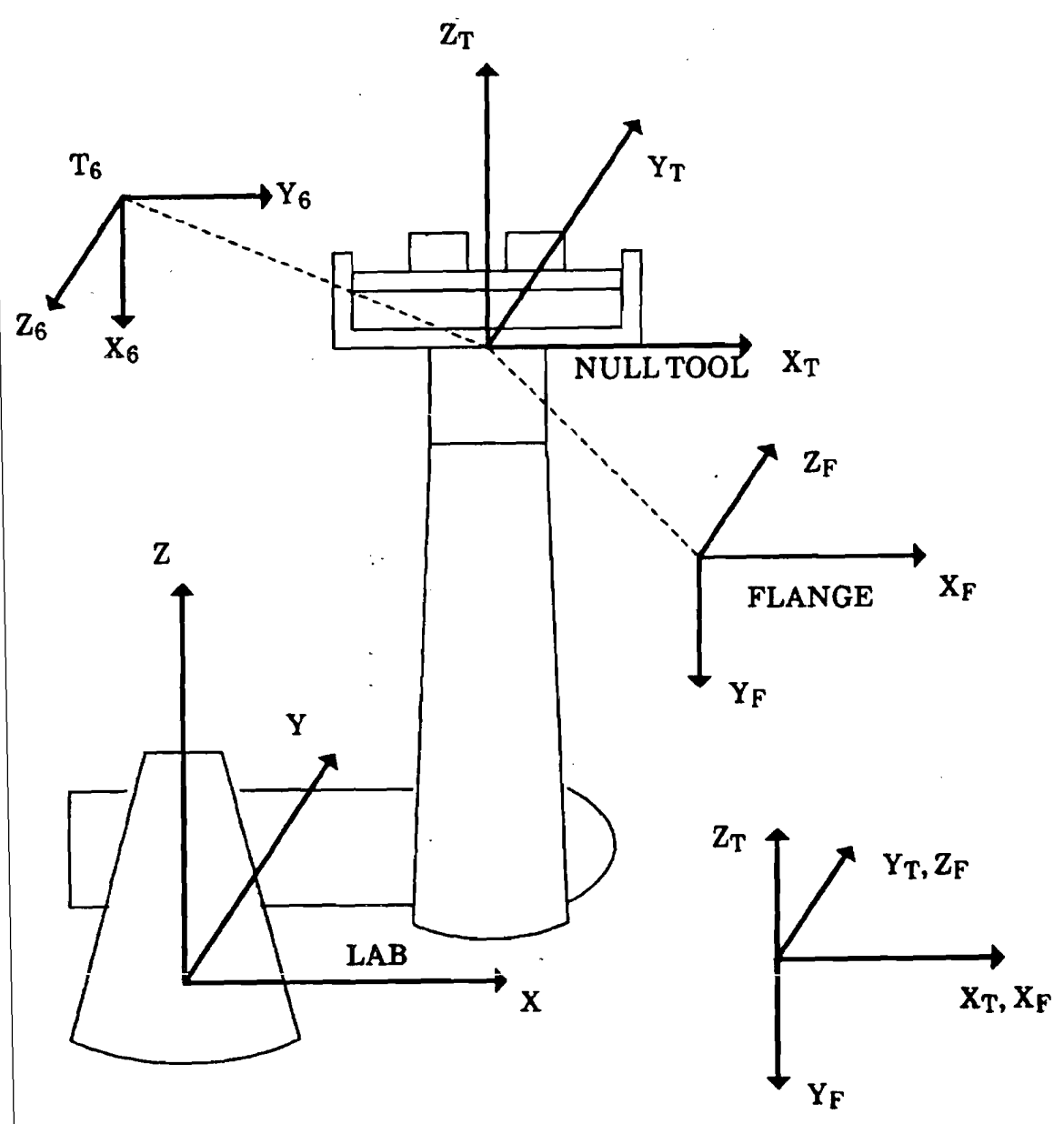

Figure 1: The Puma and head, as seen from the observation window, showing three basic CSs. The origins of $T_{6}$, NULLTOOL and FLANGE coincide. The robot is shown with JntAng $=\overrightarrow{0}$ and Head $=\overrightarrow{0}$. In this configuration $\operatorname{Loc}=(650,190,975,90,-90,0)$. 
$(0,0,0,90,-90,0)$. The O,A,T components of Loc are angles that have the following semantics. "Rotate by $-\mathrm{O}$ around (the current) $\mathrm{X}$, then by $\mathrm{A}$ around the new $\mathrm{Y}$, then by $\mathrm{T}$ around the new $Z "$. Thus they have the same flavor as Euler angles. It is easy to verify that applying the NULLTOOL transform to $T_{6}$ at JntAng $=\overrightarrow{0}$ transforms $T_{6}$ to have axes parallel to LAB.

One interesting and useful aspect of TOOL is that it can be redefined by the user. For instance, one can redefine tool as a remote point, such as a world point that is currently in view. Then it is possible to issue VAL commands that rotate the robot head around the TOOL origin. The effect is for the head to move in space and to be continuously reoriented by the robot wrist (not the camera motors) so that the cameras remain pointed at the same three-dimensional scene point.

\subsection{FLANGE}

The head is rigidly attached to the sixth robot link, and hence to $T_{6}$ and TOOL. When the eyes are facing "forward" (Head $=\overrightarrow{0})$, FLANGE is a coordinate system whose axes are oriented to be consistent with the camera imaging model (Fig. 1). In FLANGE, $\mathrm{Z}$ is out along the direction the head is facing (parallel to the optic axis of cameras if Head $=\overrightarrow{0}$ ). $Y$ is down, increasing with the row number addresses of pixels in an image, and $\mathrm{X}$ is "to the right", increasing with the column numbers of pixel addresses in the image. One common trick is to define TOOL as FLANGE. This renders the explicit FLANGE transform unnecessary. If the TOOL transform is set to $(X, Y, Z, O, A, T)=$ $(0,0,0,-180,0,-90)$, then $T_{6}$ is transformed to FLANGE by the TOOL transform within the Puma.

\section{Camera Imaging Model}

Here we are concerned with the "intrinsic" camera parameters [Tsai 1986], which govern its optical properties. "Extrinsic" properties define its location in space, and determine the CamPos coordinate system. These properties are determined by the kinematic issues discussed below. For intrinsic camera properties we use a pinhole model (e.g. [Duda and Hart 1973]), which is to say we do not correct for radial lens distortions. This is not a policy, it is just that we have not yet been motivated to do so.

The camera optic axis is out along the positive $Z$ axis. Looking out along the camera's line of sight, Y points down, increasing as does the scan-line number in the camera's image. $X$ point to the right, increasing as the pixel number along a scan line. $\mathrm{X}, \mathrm{Y}, \mathrm{Z}$ form a right-handed coordinate system. We assume the origin of coordinates is at the camera's front principal point, and that the image is formed at a distance $f$ in front of the origin in the $X-Y$ plane by point projection. Then a scene point $\vec{x}$ yields the image point coordinates

$$
(\hat{x}, \hat{y})=\left(\frac{x f}{z}, \frac{y s f}{z}\right),
$$

where $f$ is the effective focal length of the entire imaging, transmission, digitization, and ROIStoring process, and $s$ is a scaling constant that expresses the "aspect ratio" of the system. The angular (spatial) resolution of the final pixels resting in ROI is less in the $\mathrm{Y}$ direction, and $s$ tells by how much. Thus the model tells where a point appears (under default setings) in ROI store, and under default settings where its location is reported by 
FeatureMax. It includes all effects induced by CCD chip layout, conversion to analog waveform by the Panasonic electronics, sampling by DigiMax, and storage in ROI. It does not pretend to say anything about any of these effects in isolation.

The parameters $f$ and $s$ were estimated using a calibration chart, and the values in the "Constants" section represent our best current estimates.

The camera transform can be expressed as multiplying the homogeneous scene point vector by a transform matrix $C$ and then performing normalization [Tsai 86]. The normalization operation scales a homogeneous 4 -vector $(x, y, z, w)^{T}$ by $(1 / w)$. In this context, the resulting value of $\mathrm{z}$ is an artifact, since the image has only two dimensions. The matrix $\mathrm{C}$ is

$$
C=\left[\begin{array}{cccc}
f & 0 & 0 & 0 \\
0 & f s & 0 & 0 \\
0 & 0 & f & 0 \\
0 & 0 & 1 & 0
\end{array}\right]
$$

The camera extrinsic properties are determined by the LAB-Camera kinematic chain discussed next.

\section{The LAB-Camera Kinematic Chain}

There are some fourteen identifiable transforms between LAB and a CamPos Coordinate System (Table 1). The head transforms can be collapsed into two link transforms involving offsets and one rotation each [Paul 81], but in this treatment all the transforms beyond $A_{8}$ are pure rotations or translations. The Joint 1-6 transforms $A_{1}-A_{6}$ generally involve both offsets and rotations.

Define $T_{i}$ as $A_{1} A_{2} \cdots A_{i}$. These transforms, their partial products, and the inverses of their partial products, are of use in everyday robotic life. For instance, $T_{8}$ converts points expressed in FLANGE coordinates (often the output of vision routines is in FLANGE) into LAB. As another example, to simulate making an image with a camera, a point in LAB must be transformed by $\mathrm{T}_{14}^{-1}$ in order for the camera imaging model to apply.

Note that $T_{7}$ is implemented internally in VAL. We can only ask or set the value of $T_{7}$, (not $A_{1}-A_{7}$ ). $A_{8}-A_{14}$ are transforms that are created and manipulated by the user. Thus we can describe $A_{7}$ and $A_{8}$ as follows.

$\mathrm{A}_{7}$

NULLTOOL or set by user.

$\mathrm{A}_{8}$

Rot_ $x(-90)$ if TOOL $=$ NULLTOOL, Identity if TOOL $=$ FLANGE.

There are two CamAxis transforms, corresponding to the offsets of left and right cameras along the camera platform. CamAxisL and $\mathrm{R}$ are given special names only because they are the last head points that are rigidly affixed to FLANGE. Thus they may offer some efficiency for position evaluations when the eyes are moving but the head (robot) is not. From this point on there are two kinematic chains, corresponding to the differing offsets and azimuth angles of the two cameras, and denoted by $L$ and $R$. We often group $A_{8} \cdots A_{14}$ into a single transform matrix (named CamPosL or CamPosR) expressing the camera location in FLANGE coordinates. 


\begin{tabular}{|l|l|l|l|}
\hline A matrix & \multicolumn{1}{|c|}{ Name } & Const. or Var. & Resulting CS \\
\hline Ident & LAB & $\mathrm{C}$ & LAB \\
$\mathrm{A}_{1}$ & Joint 1 & $\mathrm{~V}$ & \\
$\mathrm{~A}_{2}$ & Joint 2 & $\mathrm{~V}$ & \\
$\mathrm{~A}_{3}$ & Joint 3 & $\mathrm{~V}$ & \\
$\mathrm{~A}_{4}$ & Joint 4 & $\mathrm{~V}$ & \\
$\mathrm{~A}_{5}$ & Joint 5 & $\mathrm{~V}$ & \\
$\mathrm{~A}_{6}$ & Joint 6 & $\mathrm{~V}$ & $T_{6}$ \\
$\mathrm{~A}_{7}$ & Tool & $\mathrm{V}$ & TOOL \\
$\mathrm{A}_{8}$ & FLANGE & $\mathrm{C}$ & FLANGE \\
\hline $\mathrm{A}_{9}$ & Neck Offset & $\mathrm{C}$ & \\
$\mathrm{A}_{10}$ & Eye X Offset & $\mathrm{CL}, \mathrm{CR}$ & CamAxis (L,R) \\
$\mathrm{A}_{11}$ & Altitude & $\mathrm{V}$ & \\
$\mathrm{A}_{12}$ & Alt. Offset & $\mathrm{C}$ & \\
$\mathrm{A}_{13}$ & Azimuth & $\mathrm{VL}, \mathrm{VR}$ & \\
$\mathrm{A}_{14}$ & Az. Offset & $\mathrm{C}$ & CamPos (L,R) \\
\hline
\end{tabular}

Table 1: The LAB to camera kinematic chain of transforms.

The definition of distances and angles for the robot head are shown in Fig. 2. See the section on Constants for numeric values.

\section{Forward Kinematics: CamPos from $(\phi, \theta)$}

The camera motor control software positions a camera at a specific altitude, or pitch $(\phi)$ and azimuth, or yaw $(\theta)$. We should like to compute CamPos from altitude and azimuth. CamPos is expressed in FLANGE coordinates.

$$
\text { CamPos }=\mathrm{A}_{9} \mathrm{~A}_{10} \cdots \mathrm{A}_{14} \text {. }
$$

The values of the relevant $A$ matrices are as follows (Fig. 2). Constant values are given in Section 13.

$\begin{array}{ll}A_{9} & \text { Trans }\left(0, N E C K \_O F F S E T, 0\right) . \\ A_{10} & \text { Trans(LEFT_OFFSET,0,0) or Trans(RIGHT_OFFSET,0,0). } \\ A_{11} & \text { Rot_x }(\phi) . \\ A_{12} & \operatorname{Trans}\left(0, A L T \_O F F S E T, 0\right) . \\ A_{13} & \text { Rot_y }\left(\theta_{L}\right) \text { or Rot_y }\left(\theta_{R}\right) . \\ A_{14} & \text { Trans }(0,0, \text { AZ_OFFSET). }\end{array}$

\section{Forward Kinematics: TOOL from Loc}

VAL provides several useful conversions. A "precision point" is a JntAng vector, and VAL understands robot locations in both precision points and locations (Loc 


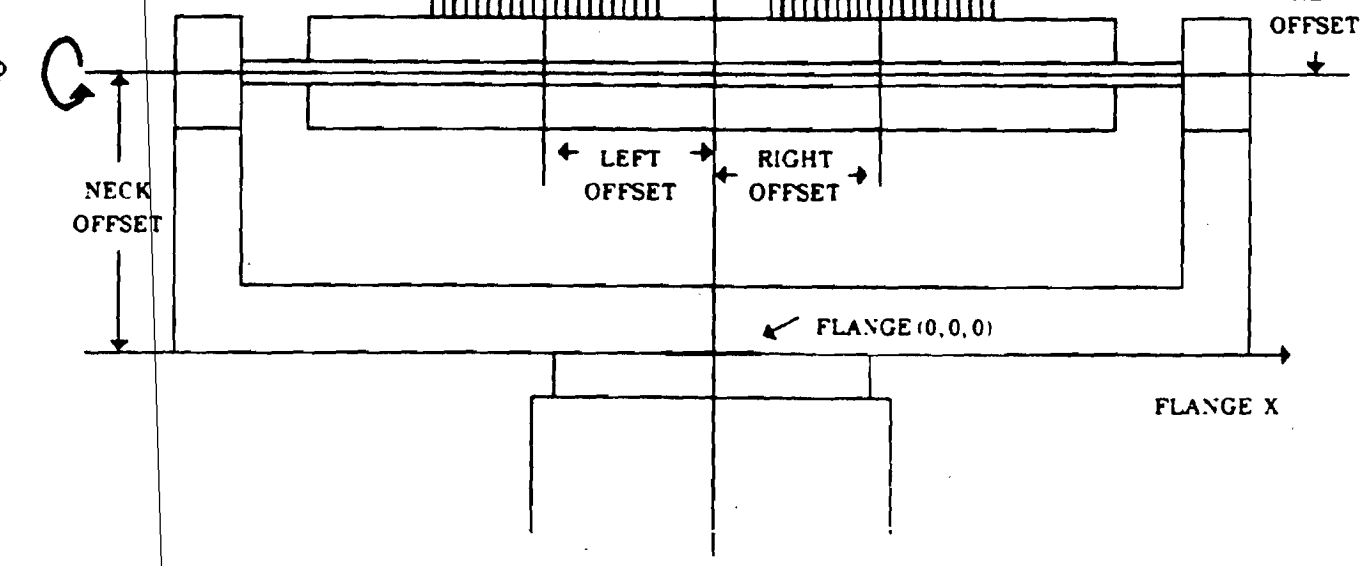

Figure 2: Axes and link offsets in the robot head (see Section 13).

vectors). It of course is possible to derive $(\mathrm{O}, \mathrm{A}, \mathrm{T})$ simply by composing all the joint angle rotations. Deriving $X, Y, Z$ from the joint angles involves knowing offsets and doing a full forward kinematics solution. Generally then the TOOL location is most easily obtained from VAL. VAL reports the TOOL location as a JntAng vector (a VAL "precision point") and a Loc vector (a VAL "location"). Our current Purdue robot control software only returns a Loc, although that is subject to change.

Converting the $(\mathrm{O}, \mathrm{A}, \mathrm{T})$ angles to a transform involves knowing exactly what they mean. The Puma manual is not explicit here. Ray Rimey determined the following transformation, which, in its alias aspect, moves LAB to the current TOOL CS.

$\operatorname{TOOL}(\operatorname{Loc})=$ Rot_z(-90)Rot_y(90)Rot_x(-O)Rot_y(A)Rot_z(T)Trans $(X, Y, Z)$.

This transformation, in its alibi aspect, thus converts points from TOOL to LAB coordinates. It is written out explicitly below. If the TOOL transform is redefined by the user, then it is to that redefined CS that LAB will be transformed. Redefining TOOL with a VAL command means setting the values of $X, Y, Z, O, A, T$ in the above transformation. Thus if TOOL is redefined within VAL from NULLTOOL to FLANGE, 
then $A_{8}$ should be the identity transform, and can vanish from the kinematic chain and from the user's external calculations.

\section{Inverse Kinematics: $(\phi, \theta)$ from CamPos}

Given a camera position expressed as a CamPos transform in FLANGE coordinates, what $\theta$ and $\phi$ angles created it? To find out, write the transform

$$
E=A_{9} A_{10} \cdots A_{14} \text {, }
$$

and notice that certain individual elements of $\mathrm{E}$ contain exactly the sines and cosines of $\phi$ and $\theta$. We see

$$
\begin{aligned}
& \phi=a t a n \_2\left(\mathrm{E}_{21}, \mathrm{E}_{11}\right), \\
& \theta=a t a n \_2\left(\mathrm{E}_{02}, \mathrm{E}_{00}\right) .
\end{aligned}
$$

This is a very simple version of the work needed to get O,A,T from TOOL.

\section{Inverse Kinematics: O,A,T from TOOL}

The FRAME command in VAL takes four input vectors that describe the TOOL axis unit vectors and origin, and returns the corresponding Loc vector $(\mathrm{X}, \mathrm{Y}, \mathrm{Z}, \mathrm{O}, \mathrm{A}, \mathrm{T})$. The interesting part of this is of course deriving $(O, A, T)$ from the TOOL CS. In turn, this is an operation quite closely related to deriving Euler angles from a transform, which is an early exercise in [Paul 81].

The approach is to multiply the five matrices from the TOOL(Loc) formula (leaving out the translation) together to get a TOOL transform D. Say

$$
D=\mathrm{B}_{1} \mathrm{~B}_{2} \mathrm{~B}_{3} \mathrm{~B}_{4} \mathrm{~B}_{5} \text {. }
$$

Then, postmultiply both sides by $\mathrm{B}_{5}^{-1}$, and look for interesting relationships elementwise between the two matrices. In this case, as in Paul's solution for Euler angles, we find that we have enough information to compute $\mathrm{O}, \mathrm{A}, \mathrm{T}$ in the form of atan_2() functions, which have good properties. Proceeding to details, use the notation $S_{A}$ for $\sin (A)$, etc, substitute $A_{i}$ for $B_{i}$ in the kinematic chain equation above, and write the resulting product of the first four $B$ matrices as

$$
\mathrm{B}_{1-4}=\left[\begin{array}{cccc}
-S_{O} S_{A} & C_{O} & S_{O} C_{A} & 0 \\
C_{O} S_{A} & S_{O} & -C_{O} C_{A} & 0 \\
-C_{A} & 0 & -S_{A} & 0 \\
0 & 0 & 0 & 1
\end{array}\right] .
$$

The complete transformation is

$$
\mathrm{D}=\left[\begin{array}{cccc}
C_{O} S_{T}-S_{O} S_{A} C_{T} & C_{O} C_{T}+S_{O} S_{A} S_{T} & S_{O} C_{A} & 0 \\
S_{O} S_{T}+C_{O} S_{A} C_{T} & S_{O} C_{T}-C_{O} S_{A} S_{T} & -C_{O} C_{A} & 0 \\
-C_{A} C_{T} & C_{A} S_{T} & -S_{A} & 0 \\
0 & 0 & 0 & 1
\end{array}\right]
$$

Section 7 establishes that $\mathrm{B}_{1-4}=\mathrm{DB}_{5}^{-1}=\mathrm{D}$ Rot_z $(-\mathrm{T})$. Performing the formal multiplication on the right hand side yields a $4 \times 4$ matrix whose elements are functions of 
the elements of D (which we know), $C_{T}$, and $S_{T}$. Equating these elements to those of $B_{1-4}$, we find our first interesting equation:

$$
S_{T} \dot{D}_{20}+C_{T} D_{21}=0,
$$

which implies

$$
T=\text { atan_ } 2\left(D_{21}, D_{20}\right) \text {. }
$$

We can also read off that

and

$$
-S_{A}=D_{22}
$$$$
-C_{A}=C_{T} D_{20}-S_{T} D_{21} \text {, }
$$

so

$$
A=a t a n_{-} 2\left(-D_{22}, S_{T} D_{21}-C_{T} D_{20}\right) .
$$

Finally, we can read off expressions for $C_{O}$ and $S_{O}$ to get

$$
\left.O=\text { atan_2( } S_{T} D_{10}+C_{T} D_{11}, S_{T} D_{00}+C_{T} D_{01}\right) \text {. }
$$

At the time of writing, these derivations have not been checked against the output of the FRAME command.

10. Inverse Kinematics: $(\phi, \theta)$ from $(x, y, z)$

Given a point $\vec{x}$ at $(x, y, z, 1)^{T}$ in FLANGE, which $\phi$ and $\theta$ parameters will center the point in a camera's view? Following the strategy of the last section did not immediately lead to a promising set of equations. The hope was that the camera physical transform $\mathrm{E}$ could be written out and the fact that $E \vec{x}=(0,0, z, 1)^{T}$ would lead to something simple. It did not seem to. Instead we use a straightforward geometric approach (Fig. 3).

From Fig. 3, we have

$$
\begin{gathered}
h=\left(z^{2}+y^{2}\right)^{1 / 2}, \\
b=a \sin (d / h), \\
\phi=a t a n \_2(-y, z)-b .
\end{gathered}
$$

The asin() is bad practice because of its ambiguity and lack of differentiation for angles near 90, but for small angles, as will usually be the case in such a setup as this, it behaves reasonably.

It remains to determine the azimuthal rotation. Rotate space by Rot_x $(-\phi)$, bringing the cameras and point into a plane of constant $y$. The point's new $(x, z)$ coordinates become $(x, z \cos (\phi)$-ysin $(\phi))$, and finally we have

$$
\theta=a t a n_{-} 2(x, z \cos (\phi)-y \sin (\phi)) .
$$




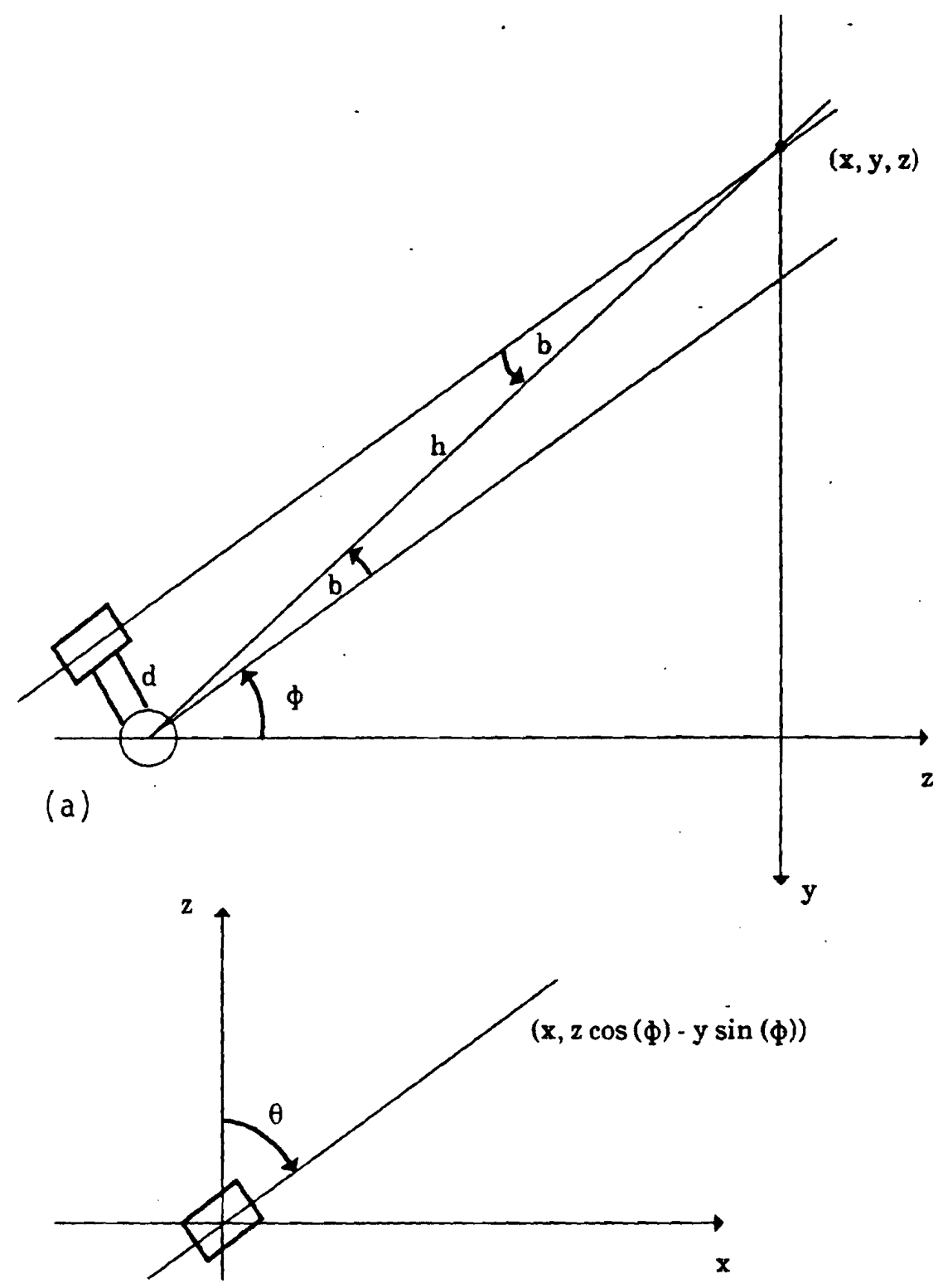

(b)

Figure 3: (a) Distances and Angles for computing the $\phi$ to aim camera at a point $\vec{x}$. (b) Distances to compute $\theta$ to aim camera at $\vec{x}$.

\section{Inverse Optics: $(x, y, z)$ from Two Images via Pseudoinverse}

The complete imaging model is

$$
(\hat{x}, \hat{y}, \hat{z}, 1)^{T}=\mathrm{P}(\text { norm }(\mathrm{CT} \vec{x})),
$$

where $\mathrm{P}$ is the PhysPixel transform that shifts the origin of pixel coordinates to the upper 
left corner from the center of the image, norm () is the homogeneous vector normalizing operation, $\mathrm{C}$ is the imaging matrix given above in the Camera Model section, and $\mathrm{T}$ is the inverse of the transform that locates the camera in LAB coordinates, i.e. it is $(F L A N G E \cdot C a m P o s)^{-1}$. First, let

$$
(\tilde{x}, \tilde{y}, \tilde{z}, 1)^{T}=\mathrm{P}^{-1}(\hat{x}, \hat{y}, \hat{z}, 1),
$$

(In our system this just amounts to defining the new variables $\tilde{x}=\hat{x}-255, \tilde{y}=\hat{y}-255$ ). Then from the definition of norm () , and letting $\vec{x}=(x, y, z, 1)^{T}$ we have

$$
(\tilde{x}, \tilde{y})=\left(\frac{[C T]_{0} \vec{x}}{[C T]_{3} \vec{x}}, \frac{[C T]_{1} \vec{x}}{[C]_{3} \vec{x}}\right),
$$

where $[\mathrm{CT}]_{0}$ is the first row of $\mathrm{CT}$, etc. Moving the denominators over to the left gives us

$$
\begin{aligned}
& \tilde{x}[\mathrm{CT}]_{3} \vec{x}=[\mathrm{CT}]_{0} \vec{x} \\
& \tilde{y}[\mathrm{CT}]_{3} \vec{x}=[\mathrm{CT}]_{1} \vec{x},
\end{aligned}
$$

and multiplying everything out and rearranging gives two linear equations in $\mathrm{x}, \mathrm{y}$, and $\mathrm{z}$ in terms of the known quantities $f$ the effective focal length, $s$ the aspect ratio, and $\mathrm{T}_{i j}$.

$$
\begin{gathered}
x\left(\tilde{x} \mathrm{~T}_{20}-f \mathrm{~T}_{00}\right)+y\left(\tilde{x} \mathrm{~T}_{21}-f \mathrm{~T}_{01}\right)+z\left(\tilde{x} \mathrm{~T}_{22}-f \mathrm{~T}_{02}\right)=f \mathrm{~T}_{03}-\tilde{x} \mathrm{~T}_{23} \\
x\left(\tilde{y} \mathrm{~T}_{20}-f s \mathrm{~T}_{10}\right)+y\left(\tilde{y} \mathrm{~T}_{21}-f s \mathrm{~T}_{11}\right)+z\left(\tilde{y} \mathrm{~T}_{22}-f s \mathrm{~T}_{12}\right)=f s \mathrm{~T}_{13}-\tilde{y} \mathrm{~T}_{23} .
\end{gathered}
$$

Thus knowing the physical locations of two cameras, and knowing the pixel coordinates of the corresponding two images of the same three-dimensional point $\vec{x}$ yields four equations in the three unknowns $(x, y, z)$. They can be solved by a pseudo-inverse method. If $\mathrm{X}$ is the matrix of coefficients of $(\mathrm{x}, \mathrm{y}, \mathrm{z})$ in the above equation and $\mathrm{Y}$ is the row matrix of the right hand sides, then the four equations can be written

$$
\mathrm{Y}=\mathrm{XB}
$$

if $\mathrm{B}$ is the formal column-vector of the variables $(x, y, z)^{T}$. The values of $\mathrm{x}, \mathrm{y}$, and $\mathrm{z}$ are obtained simply by computing the pseudo inverse of $X$ :

$$
\mathrm{B}=\left(\mathrm{X}^{T} \mathrm{X}\right)^{-1} \mathrm{X}^{T} \mathrm{Y} \text {. }
$$

The physical interpretation of this method is made difficult by the fact that the "observables" (the $\hat{x}$ and $\hat{y}$ ) and the "independent variables" (the $\mathrm{T}_{i j}$ ) contribute to coefficients of both the B matrix and $\mathrm{Y}$ vector. Analysis shows that the effect of noise on this method may be significant, since a one-pixel error in $\hat{x}$ position causes a $20 \mathrm{~mm}$ depth error at a two meter distance, and a one degree error in azimuth produces a $20 \mathrm{~mm}$ error in $x$ location. The method has been implemented and integrated into a system that obtains three-dimensional position and verifies it by touching the object with a pointer, and seems to perform as well as the more geometrically intuitive method given in the next section. One potential advantage of the pseudoinverse method is its straightforward extension to more data points. 


\section{Inverse Optics: $(x, y, z)$ from Two Images via Vectors}

Duda and Hart [1973] present a geometrically intuitive method for stereo from two image points. We have implemented it and find it works as well as the pseudoinverse method for two images. The stereo problem is posed using plain 3-vectors, not homogeneous vectors. The following vectors are defined (Fig. 4).

The two cameras have lens centers at $\vec{L}_{L}$ and $\vec{L}_{R}$. The vector $\vec{\delta}$ points from $\vec{L}_{L}$ to $\vec{L}_{R}$. The 3-D point in the scene is $\vec{x} . \vec{x}$ is imaged in cameraL as $\vec{x}_{L}=\left(\hat{x}_{L} \hat{y}_{L}\right)^{t}$, and in cameraR it is imaged into $\vec{x}_{R}$. The vector $\vec{r}_{L}$ points from the lens center of cameraL through the point $\vec{x}_{L}$ and to the 3-D point in the scene. A unit vector in the same direction is $\vec{u}_{L}$. Similarly, cameraR has $\vec{r}_{R}$ and $\vec{u}_{R}$ defined.

A temporary world coordinate system is placed at $\vec{L}_{L}$, thus $\vec{L}_{L}=\overrightarrow{0}$. (Once the 3-D point is estimated in this coordinate system, we will generally convert it to another system such as FLANGE.)

The vectors $\vec{r}_{L}$ and $\vec{r}_{R}$ are defined as follows

$$
\vec{r}_{L}=a_{L} \vec{u}_{L}
$$

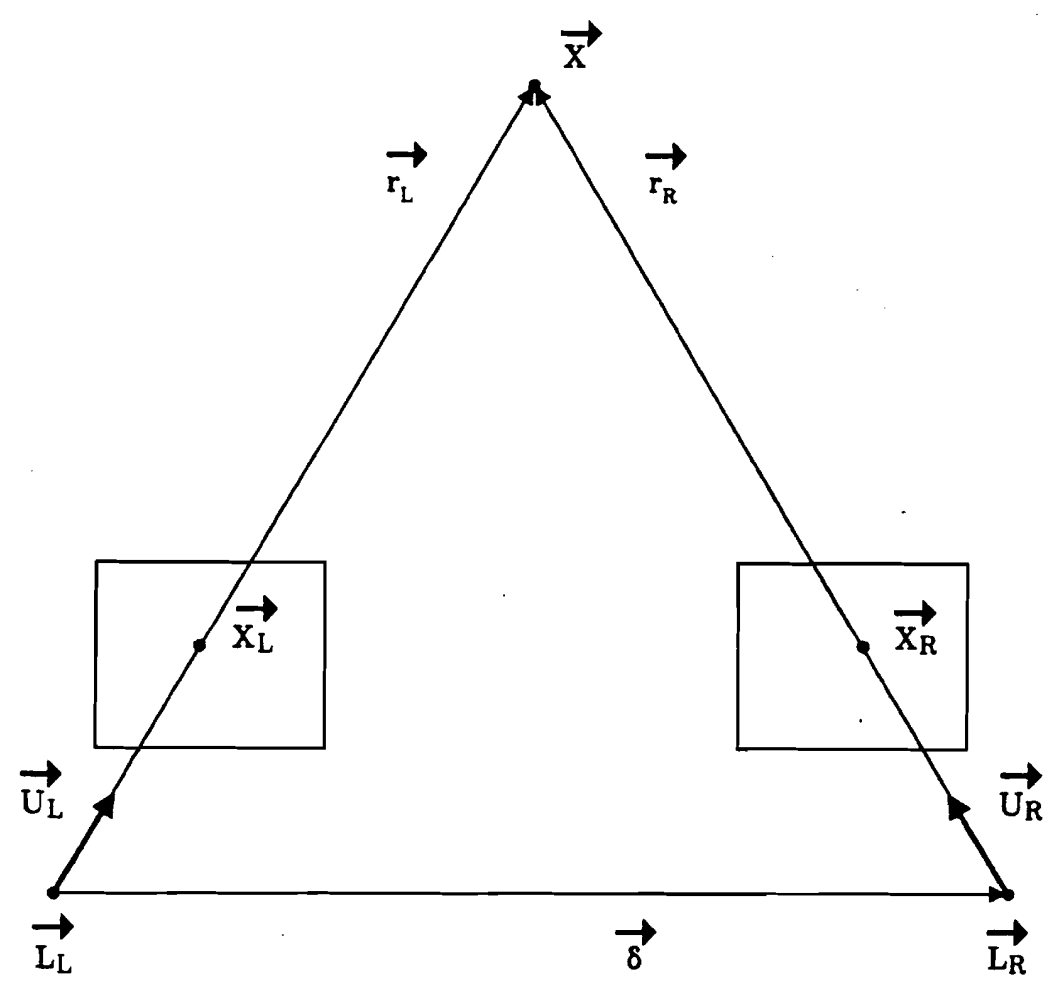

Figure 4. Vectors Used in Two-Vector Stereo Formulation. 


$$
\vec{r}_{R}=a_{R} \vec{u}_{R}+\vec{\delta}
$$

The approach is to estimate the two scalars $a_{L}$ and $a_{R}$ such that the $\vec{r}_{L}$ and $\vec{r}_{R}$ vectors are as close together as possible. Note that $\vec{\delta}$ is known and that $\vec{u}_{R}$ and $\vec{u}_{R}$ can be computed from $\vec{x}_{L}$ and $\vec{x}_{R}$. Then the value of $\vec{x}$ can be computed from $a_{L}$ and $a_{R}$ as the point midway between the heads of the $\vec{r}_{L}$ and $\vec{r}_{R}$ vectors

$$
\vec{x}=\left[a_{L} \vec{u}_{L}+\left(\vec{\delta}+a_{R} \vec{u}_{R}\right)\right] / 2
$$

The values of $a_{L}$ and $a_{R}$ are estimated by minimizing

$$
|| a_{L} \vec{u}_{L}-\left.\left(\vec{\delta}+a_{R} \vec{u}_{R}\right)\right|^{2}
$$

Duda and Hart give the answer to the minimization as

$$
\begin{aligned}
& a_{L}=\frac{+\vec{u}_{L} \cdot \vec{\delta}-\left(\vec{u}_{L} \cdot \vec{u}_{R}\right)\left(\vec{u}_{R} \cdot \vec{\delta}\right)}{1-\left(\vec{u}_{L} \cdot \vec{u}_{R}\right)^{2}} \\
& a_{R}=\frac{-\vec{u}_{R} \cdot \vec{\delta}+\left(\vec{u}_{L} \cdot \vec{u}_{R}\right)\left(\vec{u}_{L} \cdot \vec{\delta}\right)}{1-\left(\vec{u}_{L} \cdot \vec{u}_{R}\right)^{2}}
\end{aligned}
$$

A few ancilliary equations are

$$
\begin{gathered}
\vec{u}_{L}=\frac{\left(\hat{x}_{L} \hat{y}_{L} / s f\right)^{T}}{\left\|\left(\hat{x}_{L} \hat{y}_{L} / s f\right)^{T}\right\|}, \\
\vec{u}_{R}=\frac{\left(\hat{x}_{R} \hat{y}_{R} / s f\right)^{T}}{\left\|\left(\hat{x}_{R} \hat{y}_{R} / s f\right)^{T}\right\|},
\end{gathered}
$$

where $s$ is the pixel aspect ratio. Given the positions of the Left and Right cameras in FLANGE coordinates (call these CamPos transformations CamPosL and CamPosR), $\vec{\delta}$ may be computed as

$$
\vec{\delta}=\vec{L}_{R}=\operatorname{CamPosL}^{-1} \operatorname{CamPos}\left(\begin{array}{llll}
0 & 0 & 0 & 1
\end{array}\right)^{T} .
$$

If the cameras do not have parallel optic axes, then the transformation (CamPosL $L^{-1}$ CamPosR) must be applied to $\vec{u}_{R}$ to rotate it with respect to $u_{L}$.

\section{Lab Constants}

These values are taken from the directory /u/brown/robot/include, where there are several files of the form Xconsts. h.

\subsection{Head Constants \\ NECK_OFFSET \\ LEFT_OFFSET \\ RIGHT_OFFSET \\ ALT_OFFSET \\ AZ_OFFSET}

/*pitch axis to tool axis*/

$/ *$ tool $\mathrm{Z}$ axis to Left camera yaw axis*/

/*tool $\mathrm{Z}$ axis to Right camera yaw axis*/

/*am axis to pitch(altitude) axis */

$/ *$ nodal point to yaw(azimuth) axis */ 


\subsection{Camera Constants}

$\begin{array}{lll}\text { CAM_F } & 980.5 & / * \text { imaging system effective focal length*/ } \\ \text { CAM_S } & 1.289 & / * \text { imaging system pixel aspect ratio } \mathrm{y} / \mathrm{x}^{*} /\end{array}$

- $/^{*}$ the following constants allow computation of how many pixels to move for a corresponding change in angle, and vice-versa. They are accurate near image center, off by a few pixels in the periphery due to failure of small-angle assumption. The focus distance at which they are computed is the "standard focus distance " of $134 \mathrm{~cm}$, by RP and CB on 5 July. The CAM_F above also applies at this distance. */
CAM_X_P_D
17.75
CAM_Y_P_D
22.79
$/ *$ pixels per degree in $\mathrm{x} * /$
$/ *$ pixels per degree in $\mathrm{y} * /$

\subsection{Robot Constants}

$\begin{array}{ll}\text { INIT_X } & 650.0 \quad \text { *init xyzoat when all joint angles } 0 * / \\ \text { INIT_Y } & 190.13 \\ \text { INIT_Z } & 975.0 \\ \text { INIT_O } & 90.0 \\ \text { INIT_A } & (-90.0) \\ \text { INIT_T } & 0.0\end{array}$

\section{Rigid Transformation Library}

Edmond Lee wrote a library for manipulating rigid transforms as an extension to libmatrix, based on an ealier column-vector version by Dave Coombs. It provides basic and efficient implementations of standard data structures and operations. Following are exerpts from its header file.

typedef struct matrix *pt_t;

typedef struct matrix *tr_t;

pt_t pt_zero();

pt_t pt_rotx $\left({ }^{*}\right.$ pt_t p, double $\left.\mathrm{r}^{*}\right)$;

pt_t pt_roty $\left(*\right.$ pt_t p, double $\left.\mathrm{r}^{*} /\right)$;

pt_t pt_rotz $\left(/ *\right.$ pt_t p, double $\left.\mathrm{r}^{*}\right)$;

pt_t pt_translate $\left(/ *\right.$ pt_t $p$, double $\left.x, y, z^{*}\right)$;

pt_t pt_norm $(/ *$ pt_t p* $)$;

pt_t pt_transform( $\left(*\right.$ pt_t p, tr_t T, pt_t q* $\left.{ }^{*}\right)$;

tr_t tr_ident();

tr_t tr_rotx $\left(/ *\right.$ tr_t A, double $\left.r^{*} /\right)$;

tr_t tr_roty $\left(/ *\right.$ tr_t A, double r $\left.{ }^{*} /\right)$;

tr_t tr_rotz $\left(/ *\right.$ tr_t A, double $\left.r^{*}\right)$;

tr_t tr_translate $\left(/ *\right.$ tr_t $\mathrm{A}$, double $\left.\mathrm{x}, \mathrm{y}, \mathrm{z}^{*}\right)$;

tr_t tr_invert $(/ *$ tr_t $\mathrm{A}, \mathrm{B} *)$;
$/ *$ homog point $(4 \times 1$ col. vector $) * /$

$I^{*}$ homog transformation (4x4 matrix) */

/* Return new point $0001 * /$

$/ *$ sideeffects $\mathrm{p}$, rot about $\mathrm{X}$ by $\mathrm{r} *$ /

$/ *$ sideeffects $\mathrm{p}$ by normalizing it */

$/ * \mathrm{q}=\mathrm{Tp}$. returns $\mathrm{q} * /$

/* returns Identity transform */

/*sideeffects A, rot about $\mathrm{X}$ by $\mathrm{r}$ /

$/ * \mathrm{~B}$ is A inverted, B returned */ 
tr_t tr_transform $\left(/ * t_{-} \_\mathrm{t} A, \mathrm{tr}_{-} \mathrm{t} \mathrm{B}, \mathrm{tr} \_\mathrm{t} \mathrm{C} *\right) ; \quad / * \mathrm{C}=\mathrm{A} * \mathrm{~B}, \mathrm{C}$ returned */

\section{Sample Robot Data Structures and Functions}

Data structure requirements for applications vary, but the following sort of structures have been successfully used for various vision tasks, and may serve as a template for future (or possibly even standard) robot and head data structures, access functions, and update functions.

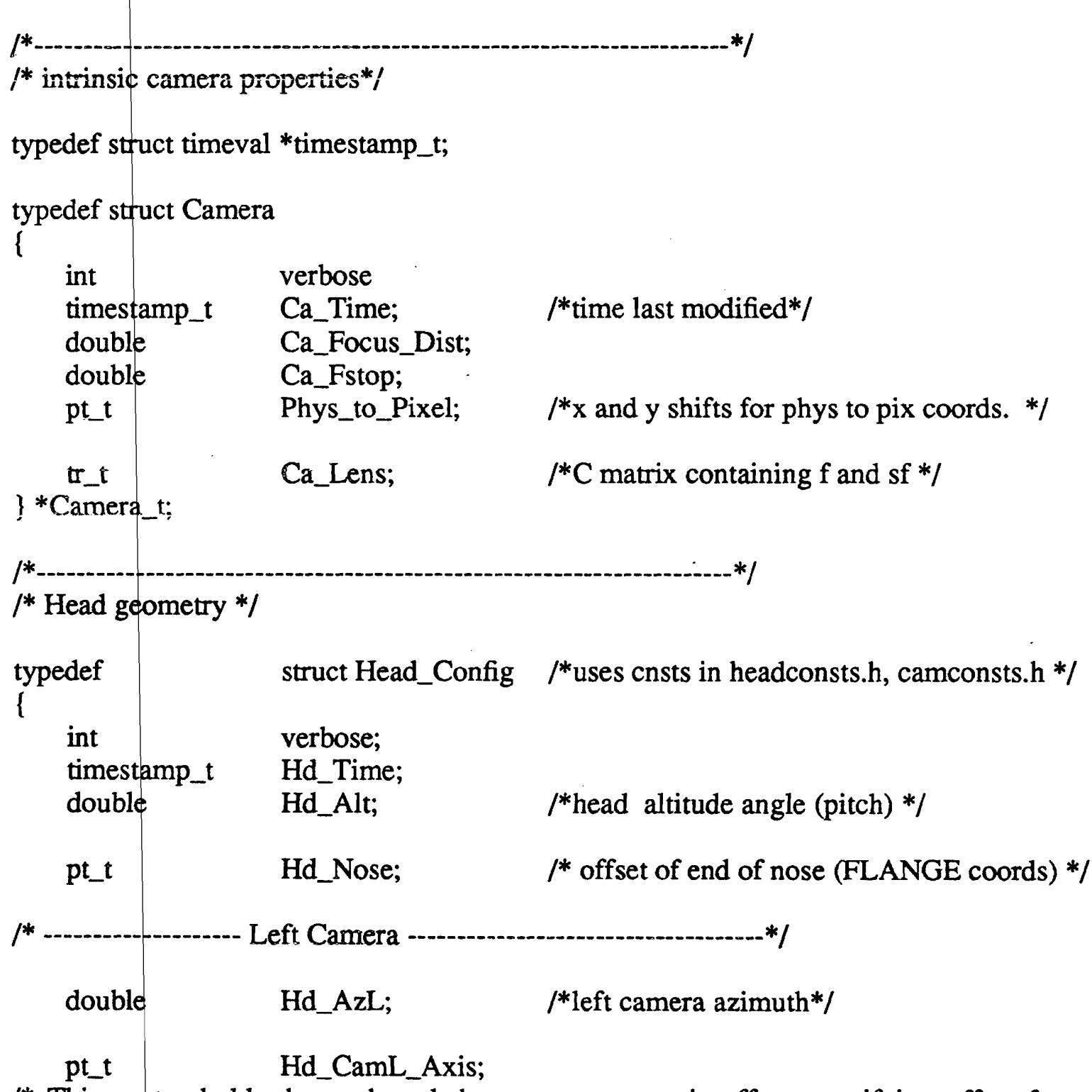

$1^{*}$ This vector holds the neck and the camera yaw axis offset, specifying offset from FLANGE origin to last rigid point in head kinematic chain. Useful intermediate transform if only eyemovements are happening. */
tr_t
Hd_CamL_Pos;
/* CamPos: camera's position in FLANGE.*/
$t r \_t$
Hd_CamL_Inv; $\quad / *$ Inverse of CamL_Pos */ 
Camera_t $\quad \cdot$ Hd_CamL;/*Left Camera state*/

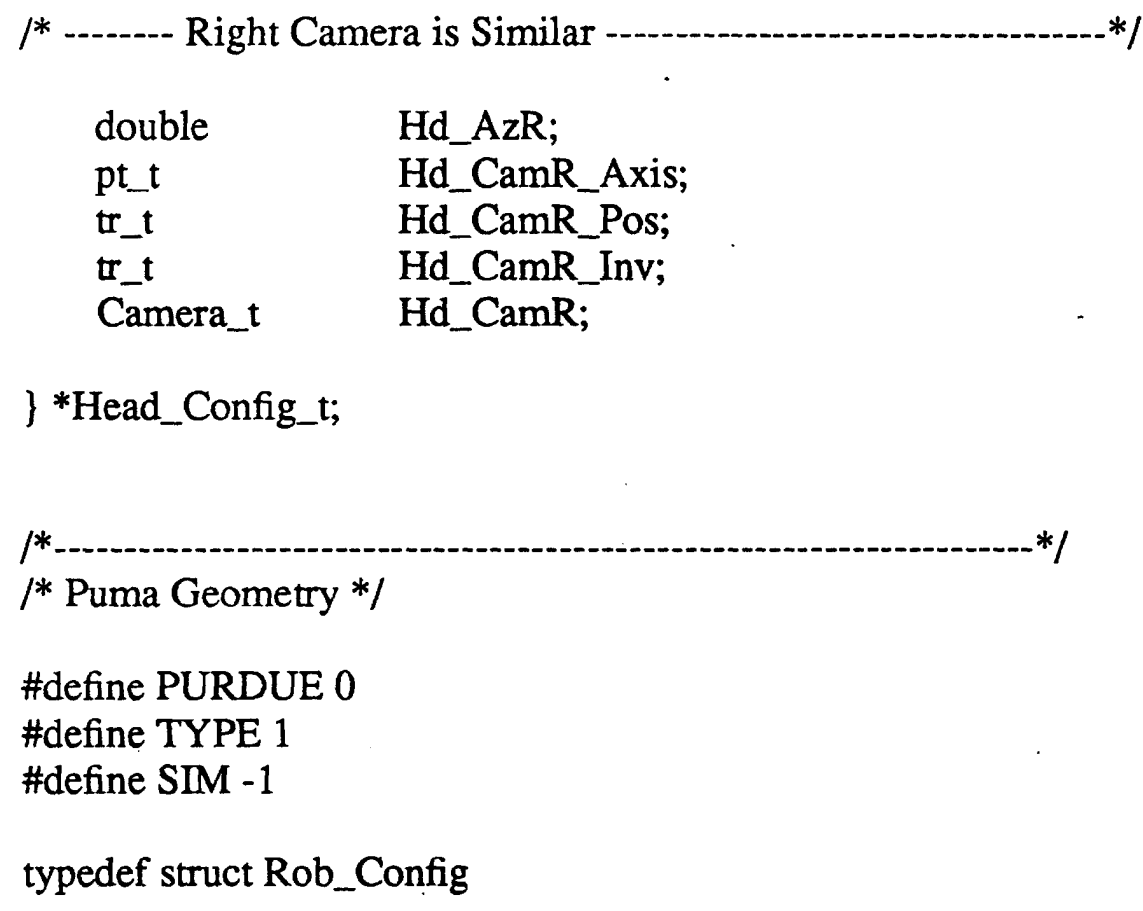

\{

$\begin{array}{ll}\text { int } & \text { verbose; } \\ \text { int } & \text { Rob_man_sim;r } \quad / * \text { purdue, console, or simulated } * /\end{array}$

int Rob_ddAlvin; $/ *$ purdue puma device descriptor*/

timestamp_t Rob_Time;

double Rob_Speed[2]; $\quad / *[0]$ is speed, [1] is mode */

double Rob_Jnt[6]; $\quad / *$ Joint angles of Joints 1-6 in order*/

double Rob_Location[6];

/*Current TOOL location as X,Y,Z,O,A,T. Updated as Robot moves. */

double Rob_Tool[6];

${ }^{*}$ Current TOOL transform as $\mathrm{X}, \mathrm{Y}, \mathrm{Z}, \mathrm{O}, \mathrm{A}, \mathrm{T}$. Set by user, same as $A_{7}$, defines TOOL in relation to $T_{6}$, does not move with robot. $*$ I
tr_t
Rob_FLANGE;
/*transform to move LAB to FLANGE *
tr_t
Rob_Fl_Inv;
$/ *$ inverse of above $* /$

Head_Config_t Rob_Head; /*Head configuration struct* $/$

\} *Rob_Config_t;

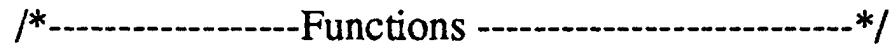

/*rob_kine.c*/ 
extern tr_t Loc_To_FLANGE $(/ *$ Loc (xyzoat) array * $/)$;

/* Returns a CS for the Rob_FLANGE entry in the Config */

extern Rob_Config_t Rob_Setup(/*simulation type*);

$/ *$ Creates Rob config, also calls head config setup */

extern void Rob_Set_Tool( $/ *$ Loc* $)$;

$/ *$ Set the TOOL CS to be that represented by Loc */

extern void Rob_Free( $/ *$ Rob_Config_t $*)$; /*destroys structure*/

extern void Rob_Move(/* Rob_Config_t, Rob_Loc */);

/*Depending on mode, moves robot or not. Updates structures. */

extern void Rob_Dump(); /*print robot state */

/*head_kine .c */

extern Head_Config_tHead_Setup();

$1 *$. Creates the head_config, puts in pre-computable tr_t's */

extern void Head_Move(/*Head_Config_t, Left_Az, Right_Az, Alt*/);

$/ *$ computes $\mathrm{L}$ and $\mathrm{R}$ Pos and Cam matrices in FLANGE coords, moves or not depending on robot mode. */

extern void Head_Free $(/ *$ Head_Config_t $* /)$;

extern void Head_Dump $(/ *$ Head_Config_t $* /)$;

extern void Cam_Dump $\left(/ *\right.$ Camera $\left.\_*\right)$;

\section{References}

Duda, Richard and Peter Hart, Pattern Classification and Scene Analysis, John-Wiley \& Sons, New York, 1973.

Paul, Richard P., Robot Manipulators: Mathematics, Programming, and Control, MIT Press, Cambridge MA 1981.

Tsai, R., "An efficient and accurate camera calibration technique for 3D machine vision", Proc. IEEE-CVPR, 364-374, 1986. (also in IEEE J. of Robotics and Automation, RA-3 4, 323-344, Aug. 1987. 